\title{
Study on joint detection of fabricated concrete shear wall based on percussion method
}

\author{
Bo Song ${ }^{1,2}$, Jian $\mathrm{Li}^{1,2, *}$, and Man sheng Wang ${ }^{1,3}$ \\ ${ }^{1}$ School of Civil and Environment Engineering, University of Science and Technology Beijing, \\ Beijing 100083, China \\ ${ }^{2}$ Earthquake Research on Earthquake Traffic Engineering in Strong Earthquake Area Beijing \\ International Science and Technology Cooperation Base, Beijing 100083, China \\ ${ }^{3}$ Beijing institute of housing and urban-rural construction science and technology, Beijing 100021, \\ China
}

\begin{abstract}
The knock detection method is one of the non-destructive testing methods for assembly prefabricated components. The knocker is used to knock on the concrete prefabricated components to be tested. The internal conditions of the tested components can be judged by analyzing the time-domain and frequency-domain diagrams of the knocker. In this paper, the impact of the change of the knock point on the detection is studied by using the knock detection experiment. It is found that the location of the knock point has a great influence on the detection. With the change of the position of the knock point, the peak frequency in the spectrum obtained by the detection changes correspondingly. According to the frequency information corresponding to the peak value in the spectrum, the depth of the void to the knock surface can be calculated.
\end{abstract}

\section{Introduction}

Assembled concrete building structure is more and more recognized by the public because of its advantages of high efficiency, high assembly speed and fast construction speed[1]. Compared with the traditional building method, the construction efficiency of assembly building can be increased by $80 \%$, and the waste rate of resources can be reduced by $70 \%$. However, due to the defects of honeycomb, hemp surface or reserved holes which are not fully filled in the fabrication or field connection engineering of prefabricated concrete buildings, the existence of these defects will seriously affect the bearing capacity and durability of components[2]. Using effective methods to detect the nature, location and scope of fabricated concrete defects, and taking corresponding measures is an important part of Engineering construction. At present, in the non-destructive testing of connection defects of concrete components, there are mainly three methods: infrared detection, ultrasonic detection and knock detection[3]. Infrared detection technology[4] absorbs the infrared radiation signal of the continuous radiation of concrete by infrared camera and converts the signal processing into the distribution of temperature field in the range of concrete to judge the internal defects and losses of concrete components, but infrared

\footnotetext{
* Corresponding author:154388870@qq.com
} 
detection technology is effective in detecting defects in deeper parts of concrete components. The fruit is bad. Ultrasound detection technology[5] has high sensitivity for the detection of the structure interior. Ultrasound can penetrate solid components and detect their interior. By judging the information of wave velocity and amplitude, we can deduce whether there are defects in concrete members, but the ultrasonic testing method is suitable for non-metallic components, and has little effect on concrete members with steel tubes reserved inside. In this paper, the knock method is used to detect the internal defects of concrete, and the factors affecting the accuracy of the knock method are explored.

\section{Tapping detection principle}

The experiment of detecting the internal vacancy of concrete by percussion method is carried out by using small steel balls lagging behind the surface of concrete blocks as vibration source and concrete blocks to be tested as propagation medium. Three kinds of waves are produced after the test block is knocked behind the ball: $\mathrm{P}$ wave parallel to the propagation direction, $\mathrm{S}$ wave perpendicular to the propagation direction, and Rayleigh wave propagating along the concrete surface, namely $\mathrm{R}$ wave.

Because of the characteristics of the fastest propagation speed and the strongest penetration ability of $\mathrm{P}$-wave, the main waveform used in the detection of concrete blocks by percussion method is $\mathrm{P}$-wave. The surface displacement caused by the stress wave produced by percussion is detected and recorded by the sensor to form a voltage-time signal, i.e. waveform. The waveform contains the frequency information of multiple one-wave reflection between the impact surface and the defect and each interface. The propagation path of $\mathrm{P}$ wave is shown in the figure.1 The displacement amplitude of concrete surface caused by $\mathrm{P}$ wave near the impact point is the largest, while that caused by $\mathrm{S}$ wave is the smallest, which is basically zero and negligible.

The measured time history curve is filtered and converted to FFT to obtain the spectrum curve for further analysis and summary.

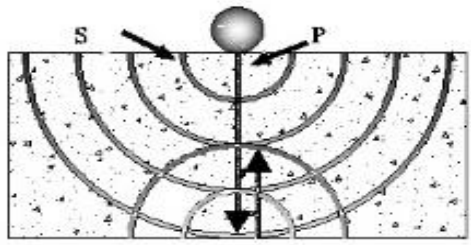

(a) P-wave and S-wave waveform schematic diagram

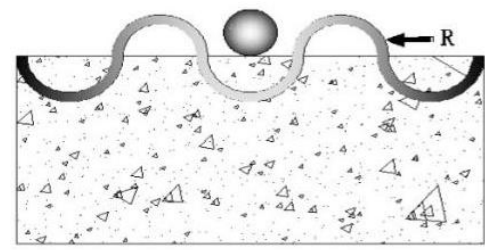

(b) Schematic diagram of $\mathrm{R}$ wave

Fig. 1. Waveform schematic diagram generated by knocking method.

\section{Experiment}

\subsection{Instrument and model}

In the experiment, the acceleration time history curve at the impact point was measured, and the frequency spectrum curve was obtained by frequency spectrum transformation (FFT), so as to judge whether the concrete block is defective or not. The instrument used in the experiment is WS-5921N network data acquisition instrument produced by Beijing Spectrum, which can quickly carry out spectrum analysis and display the refined power spectrum with cosine window in real time. It has the characteristics of fast processing speed, good automation and high measurement accuracy. 


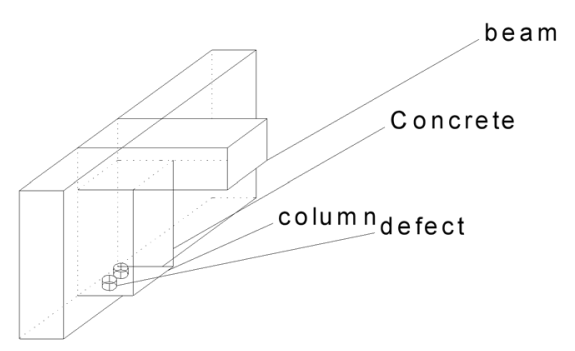

Fig. 2. Assembly structure defect diagram.

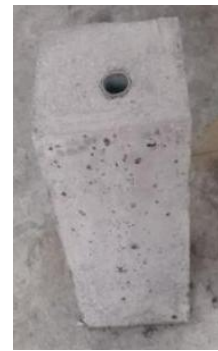

Fig. 3. Test block diagram of percussion detection.

In engineering, the grouting sleeve of assembly building structural components should be tested by joint grouting. as shown in Figure 2. In the actual testing process, the experimental model is simplified to reserve holes in concrete blocks with steel tube sleeves, as shown in Figure 3. Concrete blocks are made of cement, sand, stone and other raw materials in a certain proportion. Among them, cement is $325 \#$ Portland cement. The grain size of sand is about $0.35-0.5 \mathrm{~mm}$, the grain size of stone is about $5-20 \mathrm{~mm}$, and the mass ratio of cement, sand and stone is 1:2.89. Complete concrete blocks and defective concrete blocks with reserved voids are made respectively. Numbers 1, 2 and 3 (as shown in the figure 4) are as follows. (b) is a concrete block with reserved holes. The holes run through the cross section of the model, and the diameter of the holes is $20 \mathrm{~mm}$.

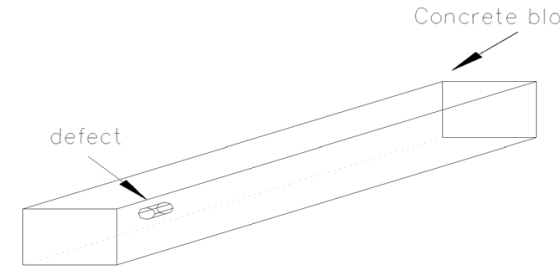

(a) Circular concrete defect blocks

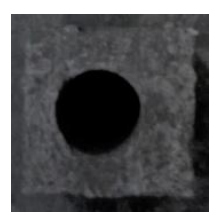

(b)concrete block with circular defect

Fig. 4. Defected concrete sketch.

\subsection{Experimental process and method.}

The three experimental models are all tested in a single channel. According to the relevant regulations of "Technical Regulations for Testing Concrete Compressive Strength by Rebound Method JGJT 23-2011"[6], the experiment should be carried out at room temperature $(5 \sim 35){ }^{\circ} \mathrm{C}$, and the hammering ball is fixed to the same height. Falling, the tapping value should take the average of the stable results of three consecutive strokes. Fixed the steel balls to the same height and fell freely. The average value of the stability results of three consecutive knocks should be taken. In order to reflect the real situation of the defects of the test blocks, eight knocking points are evenly arranged on the test surface of each concrete block, in a word arrangement, and the distance between the two adjacent points is $15 \mathrm{~mm}$.. Raw materials and matching as shown in the table 1 .

Table 1. Mix ratio.

\begin{tabular}{|c|c|c|c|c|}
\hline material & cement & water & Sand & Stone \\
\hline proportion & 1.75 & 1 & 3.96 & 5.05 \\
\hline
\end{tabular}

Draw the line of the concrete specimen that has been demolded, arrange the number of measuring points as shown in the figure, test according to the numbering sequence, and record the data. The picture shows the actual test site map. The obtained test data is 
subjected to spectrum conversion processing, and after processing, combined with the calculation formula (1), the defect of the concrete can be obtained.

$$
V p=2 f T / c
$$

In the expression

$V p$ - Known wave speed test values for precast concrete members

$f$ - Concrete member thickness reflection frequency

$T$ - Concrete member thickness

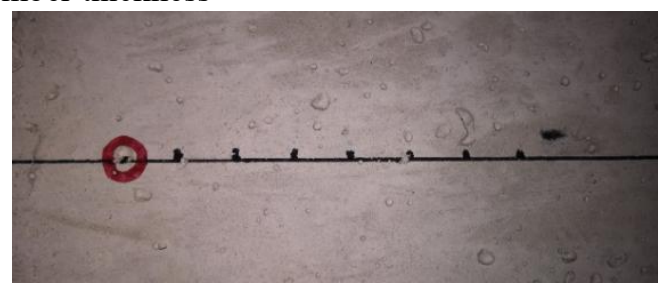

Fig. 5. Survey point layout.

The red color in the figure 5 is $0 \mathrm{~cm}$ at the time of detection, and the distance between each detection point is $1.5 \mathrm{~cm}$.

\section{Results and analysis of influencing factors}

\subsection{The influence of test point distance on the measurement}

There are many uncertain factors affecting the connection of the fabricated concrete members in the actual engineering. Since the specific location of the defects is not known before the detection, the position of the receiver and the position of the defect at the time of the tapping are caused.
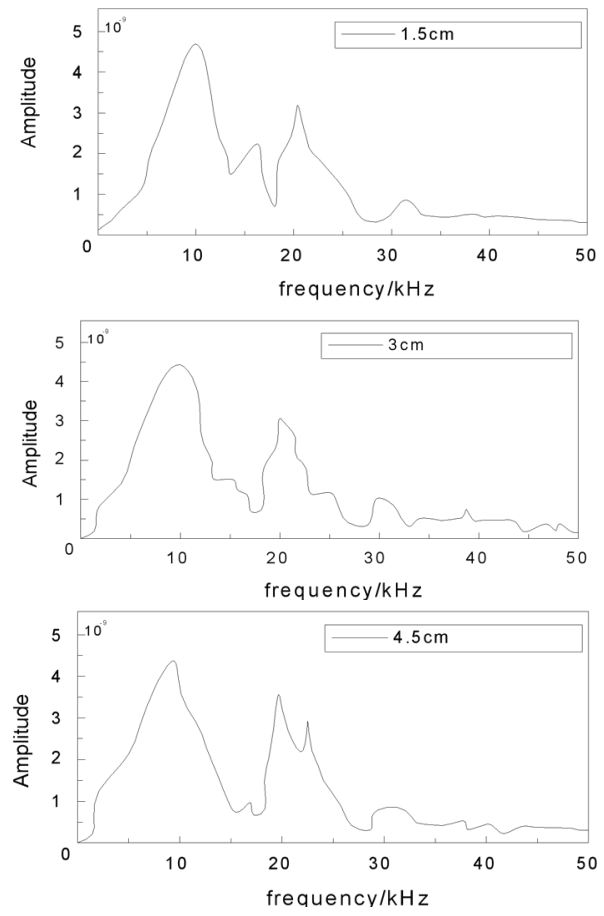

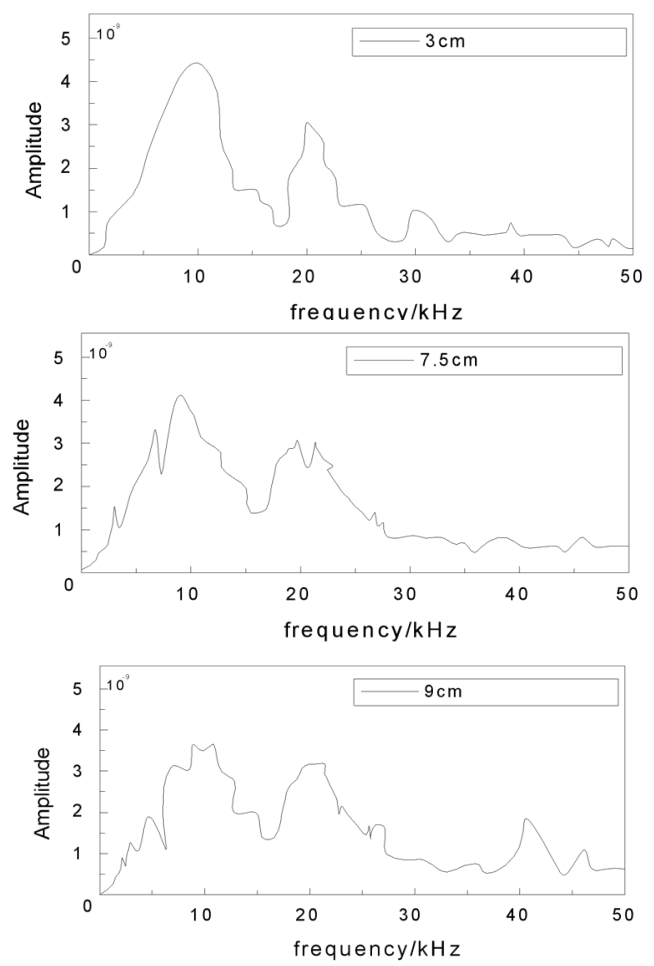

Fig. 6. Spectral map of different tapping points.

As can be seen from the above figure 6, there are 8 measuring points in the range of 1.5 $\mathrm{cm}$ to $9 \mathrm{~cm}$ in the location of the offset defect at the percussion point. With the offset of the percussion point, the amplitude curves show different spectrograms. According to the spectrum analysis after testing, the signal percussion point is more accurate between $3 \mathrm{~cm}$ and $6 \mathrm{~cm}$. There are two peaks, which are consistent with the actual situation of the test block. The reason may be that the $\mathrm{P}$ wave tested has been reflected many times in the tested block, and the internal interference and attenuation have occurred to it. After experimenting the influence of the measured point offset on the percussion test, it is found that when the percussion test is carried out, the signal percussion points should be arranged in the range of $3 \mathrm{~cm}-6 \mathrm{~cm}$.

According to the $\mathrm{P}$ wave propagation law and formula in concrete blocks, the defect detection depth is calculated when the percussion point is offset as shown in the figure 7 . It can be seen that with the offset of the received signal point, the detection error rate reaches the minimum at the offset value of $4.5 \mathrm{~cm}$. Within this range, the detection error increases gradually with the decrease of the offset distance, while in the range larger than this value, the detection error increases gradually with the increase of the offset value. It can be inferred that the signal percussion point should be placed near the range of $4.5 \mathrm{~cm}$.

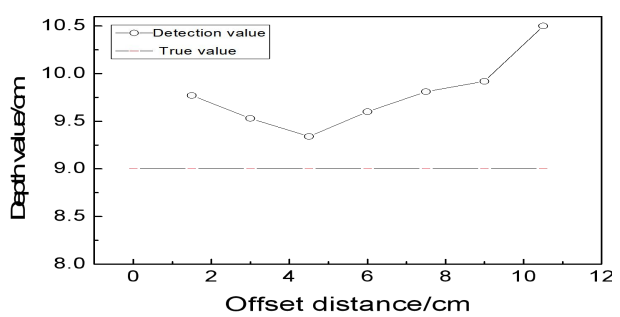

Fig. 7. Spectral map of different tapping points. 


\section{Conclusion}

The thickness of concrete blocks and the depth of internal defects can be calculated by different flat peaks in the spectrum. Through experiments, it can be concluded that the actual test thickness is slightly higher than the actual thickness of concrete blocks.

The different locations of measuring points will have some influence on the test results. With the horizontal displacement of measuring points from the defect position increasing, the error rate of the test results gradually increased. When the measuring points are located within $2-3 \mathrm{~cm}$ from the defect, the test results are more accurate. When the measuring points are located outside $3 \mathrm{~cm}$, the influence on the test results is greater.

\section{References}

1. Shao Haidong. Experimental study on testing curve of compressive strength of pumped concrete in Ningxia by rebound method [D]. Xi'an University of Architectural Science and Technology, 2012

2. Luanjian, Experimental Study on Quality Inspection of Prestressed Pipeline Grouting [D], Central South University of Forestry Science and Technology, 2011.

3. Huang Jianxin, Application of Shock-echo Method in Nondestructive Testing of Concrete Structures [D], Hehai University, 2006

4. Cheng, C.C. and M. Sansalone, Determining the minimum crack width that can be detected using the impact-echo method Part 1: Experimental study[J]. Materials \& Structures, 1995. 28(2): p. 74-82.

5. Men Jinjie et al., experimental study on acoustic emission testing parameters and stress characteristics of reinforced concrete beams [J].Journal of Xi'an University of Architectural Science and Technology (Natural Science Edition), 2015 (06): 793-798.

6. Technical Regulations for Testing Concrete Compressive Strength by Rebound Method JGJT 23-2011" 